\title{
Charaterizing The Food Habits Pattern and its Relationship With Obesity in Portugal
}

\author{
Caracterização dos Habitos Alimentares e \\ sua Relação com o Padrão de Obesidade em Portugal
}

\author{
Maria Lídia Palma, Carla Monteiro, Ana Sofia Saião \\ Escola Superior de Saúde Ribeiro Sanches (ERISA), Direcção de Farmácia - Rua do Telhal aos Olivais 8-8A, \\ 1900-693 Lisboa, Portugal, \\ E-mail: lidia.palma@erisa.pt
}

\begin{abstract}
Obesity is an endemic health problem in most of the developed countries requiring serious public health attention from authorities. In Portugal, the number of obesity cases continues to grow as suggested by the national prevalence survey published in 2007 . The present study aims to characterize the Portuguese population food pattern, and its relationship with obesity and overweight incidence. A properly validated questionnaire was applied in Lisbon area pharmacies between March and June 2010. The study included data from 452 individuals aged 18 to 92 years. Main findings show that $5.3 \%$ of the individuals were underweight (BMI <18.5), $16.6 \%$ obese (BMI between 30 and 34.9), $36.5 \%$ overweight (BMI between 25 and 29,9 ) and $41.6 \%$ normal weighted (BMI between 18.5 and 24.9). Regarding the food pattern, fish and fresh vegetables, were identified as products most conditioned by the price, a reflexion of a continuous price increase in the last years. The incidence of obesity and overweight was higher in the lower socio-economical groups, meaning that public health interventions should mainly target these disadvantaged groups which also present lower educational levels.
\end{abstract}

Keywords: Food habits; Obesity; BMI; food cost; social-economical status

\begin{abstract}
Resumo
A obesidade é um problema endémico na maioria dos paises mais desenvolvidos que requer uma maior atenção das autoridades de saúde pública. O número de casos de obesidade continua a aumentar em Portugal, como sugere o estudo de prevalência nacional publicado em 2007.0 presente trabalho tem como objectivo caracterizar os hábitos alimentares na populacao portuguesa, e relacioná-los com a incidência de obesidade e sobrepeso. Para o efeito, foi aplicado um questionario devidamente validado em farmácias da zona de Lisboa no periodo compreendido entre Março e Junho de 2010. O estudo incluiu os dados de 452 individuos com idades entre os 18 e os 92 anos. Os resultados mostram que $5,3 \%$ da amostra tinha baixo peso (IMC $<18,5$ ), 16,6\% eram obesos (IMC entre 30 e 34,9), $36,5 \%$ tinham sobrepeso (IMC entre 25 e 29,9) e 41,6\% peso normal (IMC entre 18,5 e 24,9). Quanto ao padrão de consumo de alimentos, os produtos mais condicionados pelo preço eram o peixe e os legumes frescos, grupos de alimentos que vêm sofrendo um maior aumento de preço nos últimos anos. A incidência da obesidade e sobrepeso fơ maior nos grupos sócio-económicos mais desfavorecidos, pelo que, as intervençães ao nível de saúde pública deverão ter como principal alvo os grupos de estrato sócio-económico mais desfavorecidos, e com menor nivel educacional.
\end{abstract}

Palav ras-chave: Hábitos alimentares; Obesidade; IMC; Custos dos alimentos; Estatuto sócio económico 


\section{Introduction}

Obesity is emerging in children, adolescents and adults as a pandemic. In some countries, more than half the adult population is affected, with direct consequences such as the increasing morbility and mortality of the related pathologies, specially from cardiovascular origin "II. In Portugal, two National Health Surveys, revealed that the overall overweight/obesity prevalence increased from, $49.6 \%$ (1995-1998) to $53.6 \%$ (20032005) ${ }^{\text {[]] }}$.

Several factors have been identified as a cause for the growing incidence of overweight and obesity worldwide, namely, the huge increase of food eaten per meal and related caloric input. This trend may be related to the lower cost of lower nutritional and energetic food. Also, these are normally easier to get than meat, fish, fruit and vegetables. ${ }^{[M, S]}$, Recent studies suggested that there is a higher prevalence of obesity in the lowest educated and socio-economical groups ${ }^{[7,9,9]}$. It also seems to happen the same way in Portugal, as published by Carmo etal ${ }^{[2]}$.

This study aimed to contribute to a better understanding of the relationship between the Portuguese adult population food pattern and the associated socioeconomical and educational status.

\section{Material and Methods}

\section{Participants}

A convenience, non-probabilistic sample was chosen involving pharmacy customers from the (wider) Lisboa area. These pharmacies are also educational training places for the Pharmacy students from ERISA. Individuals under the age of 18 y.o., and those showing any sort of cognitive difficulties, specially involving the meaning of the questionnaire, were not included.

This observational cross-sectional study used a questionnaire as the data collecting instrument, with closed and open questions, was operated by the pharmacy trainees and pre-tested and validated from January to February. The data collection period ran from March to June 2010, corresponding to the students training time period at the pharmacy

\section{Food patterns}

The questionnaire contained information about eventual pathology, special diets, eating out habits, and relationship between working hours and feeding habits.

\section{Introduçẫo}

A obesidade é considerada uma pandemia nas crianças, adolescentes e adultos. Mais de metade da população adulta é afectada nalguns paises, com consequências que incluem um aumento da morbilidade e mortalidade das principais doenças, sobretudo cardiovasculares, associadas ${ }^{\text {"I }}$. Em Portugal, estudos sobrea prevalência da obesidade/excesso de peso registam um aumento na dimensão deste problema, de 49,6\% (1995-1998) para $53,6 \%(2003-2005)^{2}$. Vários factores têm sido apontados como causa para o aumento da incidência da obesidade e sobrepeso a nivel mundial, designadamente, o aumento da quantidade de alimentos ingeridos por refeiçăo e o aumento das calorias ingeridas por refeiçüo. Esta tendencia podera estar relacionada com o facto dos alimentos mais energéticos e de baixo valor nutricional serem mais acessíveis, do que os alimentos de maior valor nutricional e menos energéticos, como a carne, o peixe, as frutas e os vegetais. ${ }^{[D, A]}$.

$\mathrm{Na}$ realidade, estudos recentes sugerem que existe uma maior prevalência de obesidade nos grupos socioeconómicos mais desfavorecidos e com menor grau de escolaridade ${ }^{[7, a / 9}$. Também em Portugal assim parece acontecer, como se constatou no estudo realizado por Carmo et a [?]

O presente estudo teve por objectivo contribuir para melhor conhecer a relação entre os hábitos alimentares da população adulta portuguesa, o seu estatuto sócioeconómico e grau de escolaridade.

\section{Material e Métodos}

\section{Participantes}

Foi escolhida uma amostra de conveniência, não probabilistica entre os utentes de farmácias da zona da grande Lisboa, utilizadas como locais de estágio pelos estudantes de Farmacia da Escola Superior de Saúde Ribeiro Sanches. Foram excluidos os individuos com idade inferior a 18 anos bem como aqueles que revelassem qualquer dificuldade cognitiva ou de compreensão

O presente estudo, observacional descritivo transversal, utilizou como instrumento de recolha de dados, um questionário contendo perguntas fechadas e abertas, operacionalizado pelos estagiários e previamente testado e validado durante os meses de Janeiro e Fevereiro. $O$ período de recolha de dados coincidiu com o periodo de estágio, que decorreu de Março a Junho de 2010..

\section{Hábitos alimentares}

O questionário (ANEXO 1) continha informação sobre a existencia de patologia e eventual de dieta especial, hábitos de comer fora de casa, e avaliação sobre a relação entre o horário de trabalho e os hábitos alimentares. 
Foodbuying habits

Subjects were inquired about the relationship between food prices and their buying options, which food would they choose to eliminate or to buy as a function of its own budget.

\section{Soctal-economic status}

The questionnaire contained information about the family situation, occupation, education and monthly net income.

\section{Body Mass Index (BMI)}

Each participant was asked about their age, height and weight, and the BMI calculated. Five BMI categories were considered - the first represents the population with low weight in which $\mathrm{BMI}<18.5$, normal weight is defined as BMI between 18.5 and 24.9 , overweight for BMI from 25 to 29.9 , obesity for a BMI between 30 and 34.9 and morbid obesity for $\mathrm{BMI} \geq 30^{\text {(1) }}$.

\section{Statistical analysis}

Univariate and bivariate descriptive statistics were performed with SPSS 18.0 (Microsoft). The chi-square hypothesis test was chosen and a confidence level of $95 \%$ adopted.

\section{Results}

From the 452 inquired subjects, $66.4 \%(300 / 452)$ were female and $33.6 \%(152 / 452)$ male, aged 19 to 92 years, mean $48.365 \pm 17.53$ years.

Regarding the individual's origin, 96\% (434/452) were Caucasian and 4\% (18/452) Negroid. (Figure 1)

Regarding marital status, 23\% (104/452) were single, $58.4 \%$ (264/452) married or living with a partner and $9,3 \%(42 / 452)$ widowed or divorced (same percentage for both). (Figure 1)

In terms of scholarity, $43.6 \%(197 / 452)$ possessed the 2nd cycle or a secondary degree, $28.8 \%$ (125/452) higher education and $27.7 \%(130 / 452)$ the first degree or less. We've observed for BMI that $41.6 \%(188 / 452)$ of respondents had normal weight, $36.5 \%$ (165/452) were overweight, $15.7 \%$ (71/452) were obcse, $5.3 \%$ (24/452) underweighted and only $0.9 \%(4 / 452)$ had morbid obesity (Figure 2).

A highly significant positive correlation was found between BMI and educational level of respondents ( $p<$ 0,001) (Table 1), (Figure 3).
Hábitos de compra de alimentos

Os individuos foram inquiridos sobre a relação entre o preço dos alimentos e as suas opçōes de compra e quais os alimentos que reduzem por causa do preço e quais os que aumentariam se tivessem um orçamento maior.

\section{Estatuto sócio-económico}

O questionário continha informaçã̃o sobre a situaçâo familiar e a sua ocupação profissional, se auferia pensāo ou reforma, qual o nivel de escolaridade e qual o montante mensal liquido dos ganhos salariais.

Indice de Massa Corporal (IMC)

Os individuos foram inquiridos sobre o seu peso e altura e posteriormente foi calculado o IMC. No presente estudo foram criadas cinco categorias para o IMC: a primein representa a população com baixo peso em que $\mathrm{IMC}<18,5$; o peso normal é definido para um IMC entre 18,5 e 24,9; o sobrepeso para o IMC compreendido entre 25 e 29,9 ; a obesidade para um IMC entre 30 e 34,9 e a obesidade mórbida para o $\mathrm{IMC} \geq 30^{\mathrm{m}}$.

\section{Anólise estatística}

Utilizámos estatística descritiva univariada e bivariada, realizada através do programa SPSS 18,0 (Microsoft). Para a análise dos dados foi escolhido o teste de hipóteses Qui-quadrado utilizando um grau de confiança de $95 \%$.

\section{Resultados}

Dos 452 individuos inquiridos, 66,4\% (300/452) eram do sexo feminino e $33,6 \%$ (152/452) do sexo masculino com idades compreendidas entre os 19 e os 92 anos, sendo a média de idades de 48,365 $\pm 17,53$ anos.

No que diz respeito d sua origem, $96 \%(434 / 452)$ cram caucasóides e 4\% (18/452) negroides. (Figura 1).

Em relação ao estado civil, 23\% (104/452) eram solteiros, $58,4 \%$ (264/452) encontravam-se casados ou viviam em uniảo de facto e $9,3 \%(42 / 452)$ eram viúvos ou divorciados (percentagem igual para ambos). (Figura 1).

Em termos de escolaridade 43,6\% (197/452) possuia o $2^{\circ}$ ciclo ou Ensino Secundário, 28,8\% (125/452) o Ensino Superior e $27,7 \%(130 / 452) 1^{\circ}$ ciclo ou menos. No que diz respeito, ao IMC foi possivel verificar que $41,6 \%$ (188/452) dos inquiridos tinhem peso normal, 36,5\% (165/452) tinham sobrepeso, 15,7\% (71/452) eram obesos, 5,3\% (24/452) tinham baixo peso e apenas 0,9\% (4/452) possulam obesidade mórbida (Figura 2).

Da análise dos dados foi possível verificar a existência de uma relaçâo estatisticamente significativa entre o IMC e o grau de escolaridade dos inquiridos $\left(X^{2}=31,363\right.$; $\mathrm{p}<0,001$ )(Tabela 1, Figura 3). 


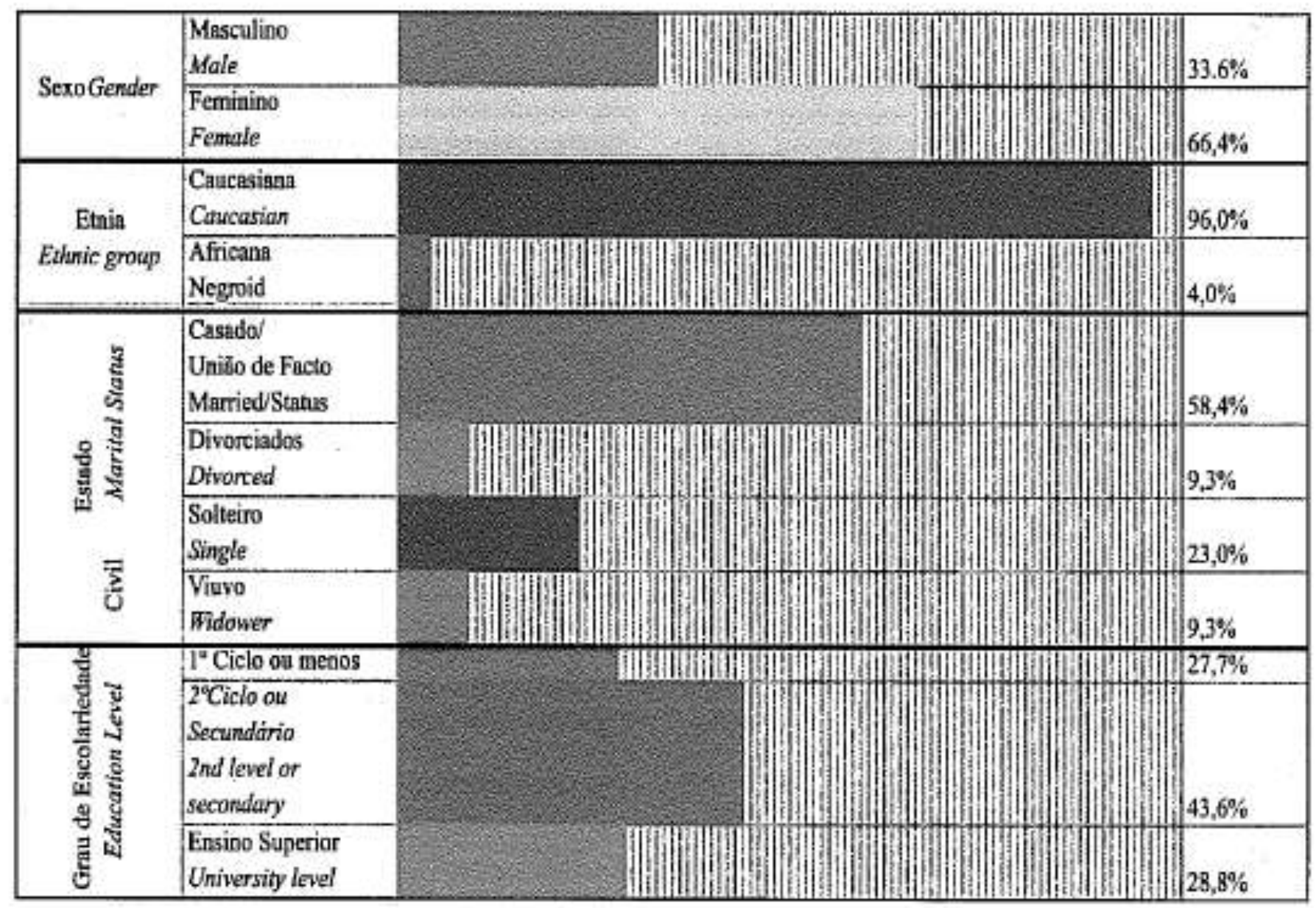

Flgure 1 - Socio-demographic sample

Figura 1 - Caracterizaçaెo Sócio-Demográfica da amostra

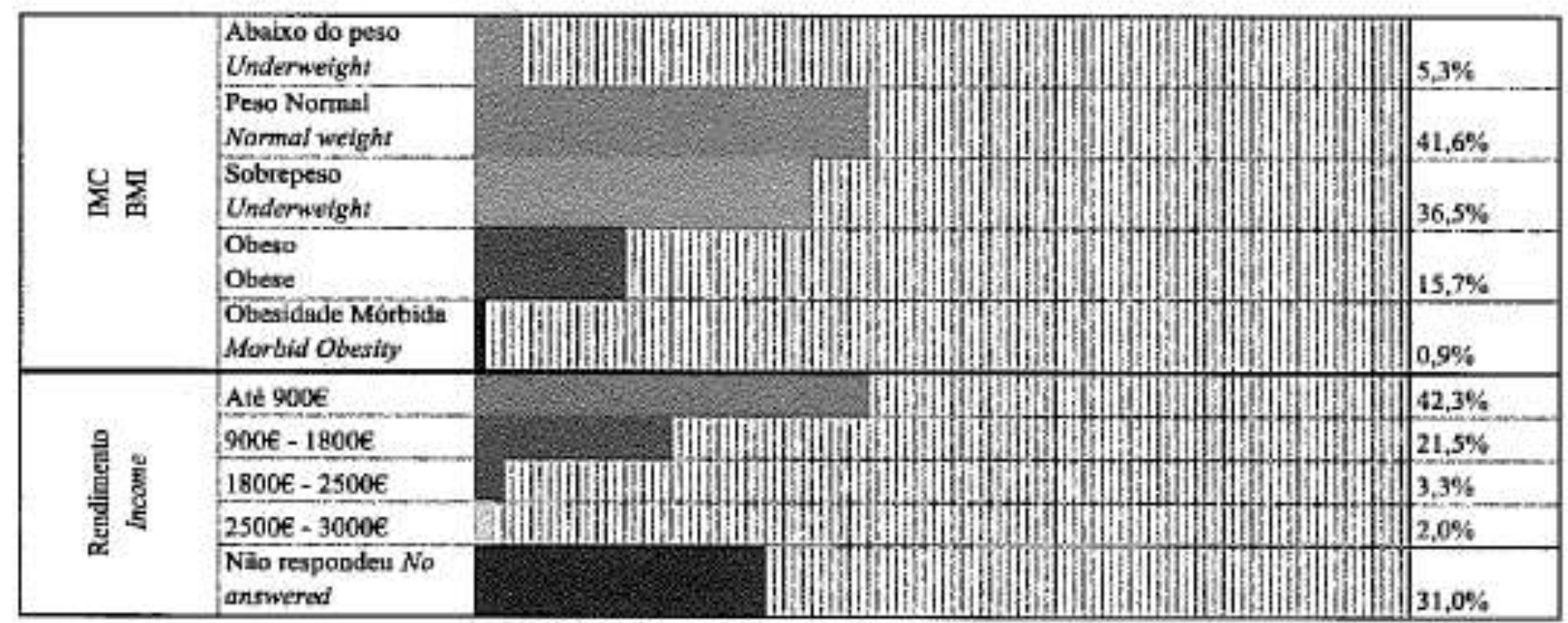

Figure 2 - Household income characterization and sample BMI

Figura 2 - Caracterizıçāo do Rendimento Familiar e IMC da amostra 


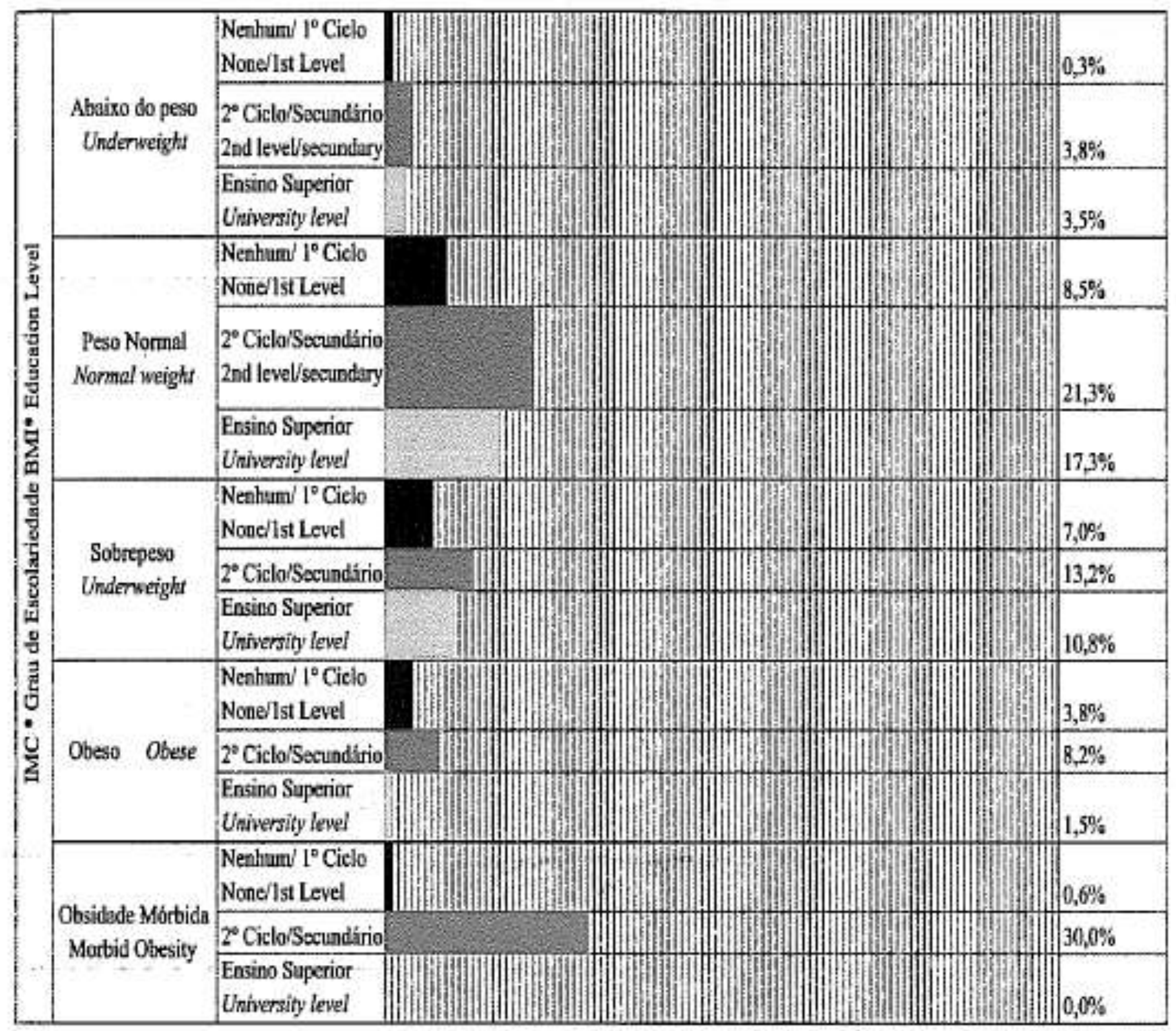

Figure 3 - BMI characterization vs education level Flgura 3 - Caracterização do IMC Vs Grus de scolaridade
Only $28.8 \%(130 / 452)$ of the responders claimed to have a special diet (Figure 4), and we confirmed that thesc were all older than 36 years and overweighted, obese or even morbid-obese. In these 130 subjects with a special diet we found that $66.9 \%(87 / 130)$ reported to suffer from some disease, being $70.8 \%(92 / 130)$ women and $29.2 \%(38 / 130)$ men. Reasons for this diet included health reasons $(63.8 \%$ : $83 / 130$ beauty $(20.8 \%$ : $27 / 130)$, overweight concerns $(9.2 \%: 12 / 130)$ for medical advise $(8.5 \%: 11 / 130)$, its own initiative $(3.1 \%$ $: 4 / 130$ ) and by the family or relative's encouragement $(2 \%: 3 / 130)$.
Dos inquiridos apenas 28,8\% (130/452) afirmou realizar uma dieta especial (Figura 4), e destes, foi possivel verificar que todos tinham idade superior a 36 anos e sobrepeso, obesidade ou mesmo obesidade mórbida. Dos 130 individuos que realizaram uma dieta especial, verificamos que $66,9 \%(87 / 130)$ afirmou sofrer de alguma patologia, dos quais $70,8 \%(92 / 130)$ eram mulheres e $29.2 \%$ (38/130) homens. No que diz respeito aos motivos da dieta $63,8 \%(83 / 130)$ dos inquiridos afirmou ser por questōes de saúde, $20.8 \%$ (27/130) por questōes de estética, 9.2\% (12/130) por excesso de peso, $8.5 \%(11 / 130)$ por conselho médico, $3.1 \%(4 / 130)$ por iniciativa própria e $2 \%(3 / 130)$ devido ao incentivo de familiares. 


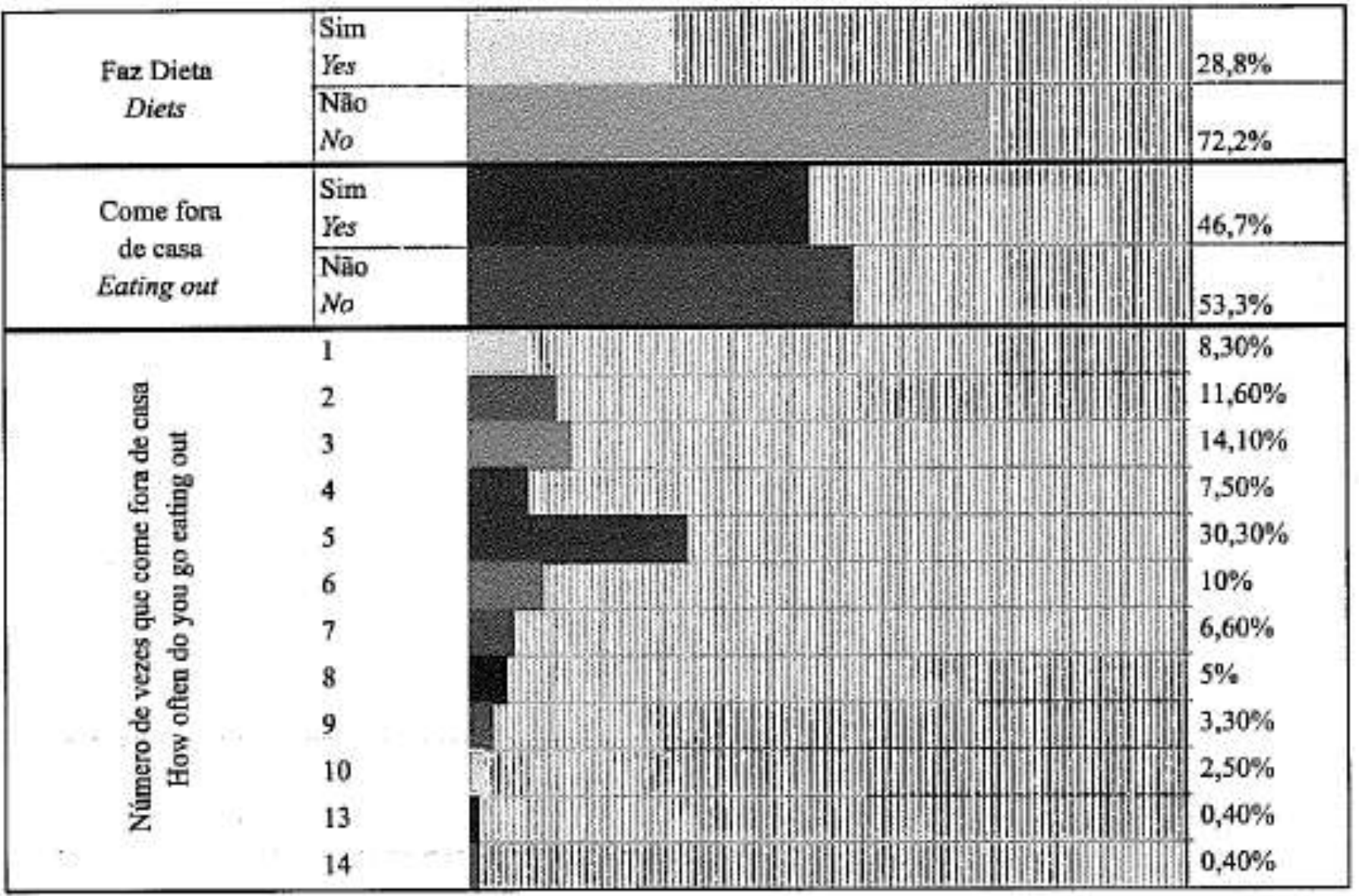

Figure 4-Food patterns

Figura 4-Caracterizaçăo dos hábitos alimentares

A highly significant positive correlation was found between between age and the pathological condition ( $p$ $<0.001$ ), with a higher incidence of pathology in older responders (Table 1 ).
Verifica-se que existe uma relação estatisticamente significativa entre a idade e a existência patologia ($\left.\mathrm{X}^{2}=96,122 ; \mathrm{p}<0,001\right)$, observando-se uma maior incidência de patologia dos inquiridos mais velhos. (Tabela 1).

Table 1 - Chi-square test, $95 \%$ significance

Tabela 1 - Teste do Qui-Quadrado com $95 \%$ de confiança

\begin{tabular}{|l|c|c|c|}
\hline & $\begin{array}{c}\text { Estatistica de Teste } \\
\text { Stot/stical Test }\end{array}$ & $\begin{array}{c}\text { Graus de Uberdade } \\
\text { Freedon Level }\end{array}$ & P-Value \\
\hline $\begin{array}{l}\text { IMC vs Grau de Escolariedade } \\
\text { BMi vs education level }\end{array}$ & 31,363 & 8 & 0,000 \\
\hline $\begin{array}{l}\text { Rendimento Vs Escolha do produto } \\
\text { Incame vs product cholce }\end{array}$ & 31,374 & 7 & 0,000 \\
\hline $\begin{array}{l}\text { Idade Vs sofre de alguma Patologia } \\
\text { Age vs pathology }\end{array}$ & 96,122 & 5 & 0,000 \\
\hline $\begin{array}{l}\text { Comer fora Vs Hordirlos de trabalho } \\
\text { Eating out vs working timetable }\end{array}$ & 42,805 & 1 & 0,000 \\
\hline
\end{tabular}


Regarding the socio-economical status the family income was an extremely important variable although difficult to answer, since $31 \%(140 / 452)$ of subjects refused to answer this question. $42.3 \%$ (191/452) of the subjects had a family income up to $€ 900,21.5 \%$ (97/452) between $€ 900$ and $€ 1800,3.3 \%$ between 1800 and $2500 €$ and $2.0 \%(9 / 452)$ between 2500 and $3000 €$ (Figure 2). A highly significant correlation was found between the household income and food choices $(\overline{\mathrm{p}}<0.001)$, (Table 1). Results showed that $52.1 \%$ (150/288) of lower income individuals had more troubles to take economical decision while $41.6 \%$ (10/24) have not referred this aspect. Analysis of the subject elimination choices as a function of price reveal fish $24.1 \%(133 / 553)$, meat $19.9 \%$ (110/553), dairy products $10.3 \%(57 / 553)$, fruit $9.0 \%(50 / 553)$ and fresh vegetables $7.8 \%(43 / 553)$. On the other hand, products referred as preferred if they just could afford them were fish $24.9 \%(191 / 768)$, meat by $18.6 \%$ (143/768), fresh vegetables $10 \%$ ( $77 / 768)$, dairy products $9.5 \%$ (73/768) and fruits $9.9 \%(76 / 768)$ (Figure 5).

The job timetable also influences meals, since $37.4 \%$ (152/452) said that his would affect their food pattern (Figure 6). A highly significant positive correlation was found between eating out and job schedules ( $p<0.001$ ), since $26.8 \%(121 / 452)$ of subjects referring this influence were eating out, while only $6.9 \%(31 / 452)$ referred to choose to eat at home (Figure 7, Table 1). We've noticed that $46.7 \%(211 / 452)$ claimed to eat out and that $53.3 \%$ (241/452) did not. In the first group, the majority $(71.8 \%: 173 / 241)$ eat out up to 5 times a week and only $3.3 \%(8 / 241)$ eat out 10 or more times a week (Figure 4). A highly significent correlation was found between eating out, family income and responder's age ( $\mathrm{p}<0.001$ ), suggesting that younger higher income individuals have mostly chosen to eat out (Table 1)
Quando analisámos o estatuto sócio-económico verificámos que o rendimento familiar foi uma variável de extrema importincia mas de dificil resposta, visto que, $31 \%$ (140/452) dos inquiridos se recusaram a responder à pergunta sobre o rendimento familiar. Dos respondentes $42,3 \%$ (191/452) possuia um rendimento farniliar até $900 €, 21,5 \%(97 / 452)$ entre $900 €$ e $1800 €$, $3,3 \%$ entre $1800 €$ e $2500 €$ e $2,0 \%$ (9/452) entre $2500 €$ e $3000 €$ (Figura 2). Da análise entre o rendimento familiar e a escolha dos alimentos verificamos uma relação estatisticamente significativa $\left(X^{2}=31,374\right.$ $\mathrm{p}=0,000$ ), entre o rendimento familiar e a escolha dos produtos alimentares. (Tabela 1) $52.1 \%$ (150/288) dos individuos com rendimentos mais baixos eram os que possuiam mais preocupaç̌es na altura de realizarem as suas compras enquanto $41.6 \%$ (10/24) dos individuos com rendimentos familiares mais elevados não tinham esta preocupaçăo.

Quando analisamos as escolhas dos inquiridos, foi possivel observar que os produtos que as familias mais reduzem nas suas compras por causa do preço são: 0 peixe $24.1 \%$ (133/553), a carne $19.9 \%$ (110/553), os lacticinios $10.3 \%(57 / 553)$, a fruta $9.0 \%(50 / 553)$ e os legumes frescos $7.8 \%$ (43/553). Por outro lado, os produtos que os inquiridos afirmam que gostavam de adquirir caso tivessem um rendimento familiar maior seriam: o peixe $24,9 \%(191 / 768)$, a carne $18.6 \%$ (143/768), os legumes frescos $10 \%(77 / 768)$, os lacticínios $9.5 \%(73 / 768)$ e as frutas $9.9 \%(76 / 768)$ (Figura 5).

O hofatirio de trabalho também influencia a realização das refeições, visto que $37,4 \%$ (152/452) afirmou que o seu horírio de trabalho influencia os seus hábitos alimentares (Figura 6). Verificou-se que existia ume relação estatisticamente significativa entre o comer fora de casa e os horários de trabalho $\left(X^{2}=63,578 ; \mathrm{p}<0.001\right)$, já que, $26.8 \%$ (121/452) dos indivíduos que afirmaram que o horário de trabalho afecta os seus hábitos alimentares eram os que comiam fora de casa, enquanto, apenas $6,9 \%$ (31/452) afirmou optar por comer em casa apesar dos horários de trabalho (Figura 7, Tabela 1). Registámos que $46,7 \%$ (211/452) afirmou comer fora de casa e que $53,3 \%$ (241/452) não recorria a esta opção. Dos primeiros, a maioria $71,8 \%(173 / 241)$ optava por comer fora de casa até 5 vezes por semana e apenas $3,3 \%(8 / 241)$ o fazia 10 ou mais vezes (Figura 4 ). Verificámos existir uma relação estatisticamente significativa entre a opção de comer fora, o rendimento familiar e a idade dos inquiridos, (em ambos $\left(X^{2}=42,805 ; p<0.001\right)$, sugestiva de que eram os individuos com um rendimento familiar mais elevado e os mais novos os que mais optam por comer fora de casa.(Tabele 1) 
Figure 5 - Food buying habits

Figura 5 - Caracterizaçūo das aquisições dos produtos alimentares

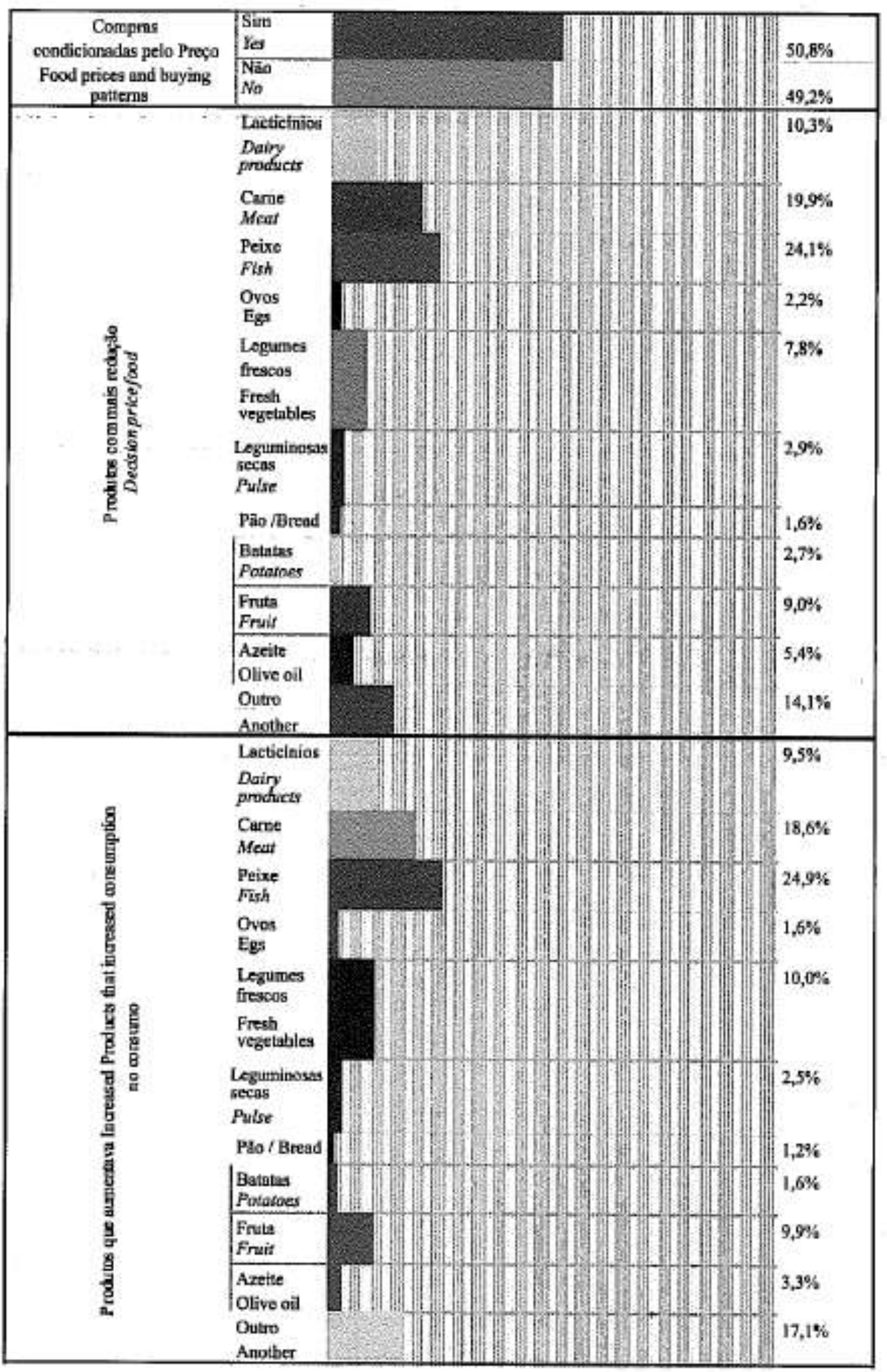




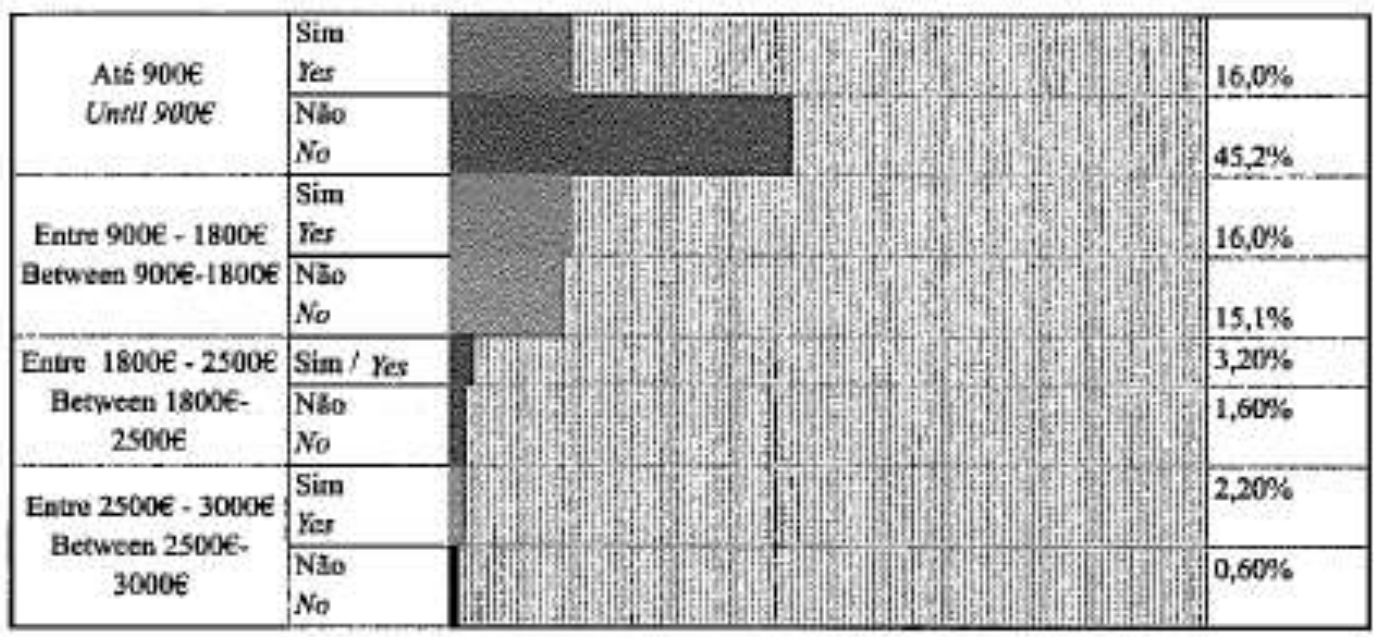

Figure 6 - Household income * Meals timetable affect food patterns

Figura 6 - Rendimento familiar ${ }^{*}$ Horário alimentar afectar os hábitos alimentares

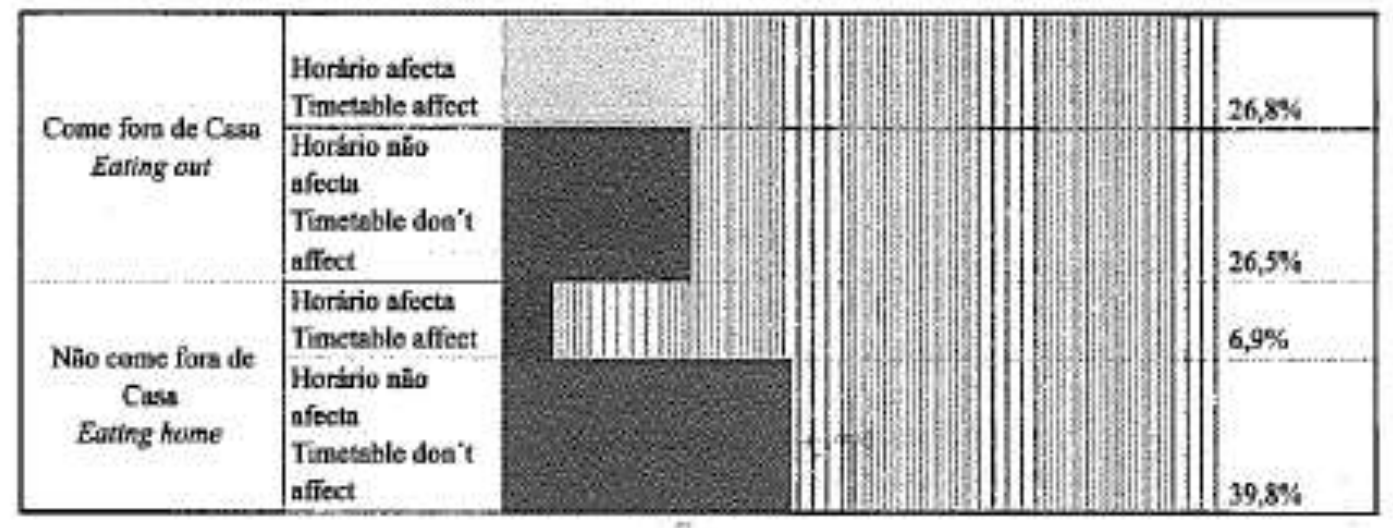

Flgure 7 - Eating out option * Working timetable affect food patterns

Figura 7 - Oppiio por comer forn de casa * $O$ borírio de trubalho afecta os hábitos alimentares

\section{Discussion}

Latest WHO data indicate that in 2008, approximately 1.5 billion overweighed adults (age $>20$ years), from which more than 500 million were obese ( 200 million men and 300 million women), existed allover the globe ii) 2015 projections indicate that by that time, 2.3 billion adults will be overweight and more than 700 million obese $^{\text {iI! }}$.

The present study resulted from a survey applied to some pharmacies in the Lisboa area, through which we have attempted to characterize consumer's food pattern, consuming habits, socio-economical status, and correlate all of these, by a multidimensional questionnaire, with the obesity prevalence. Results show that more than half of the pharmecie's consumers are obese or overweight. Detected obesity and overweight prevalence is in accordance with the latest

\section{Diseussiio}

Os últimos dados da OMS indicam que globalmente, em 2008, existiam aproximadamente 1,5 biliōes de adultos (idade 20 anos) com peso a muis, dos quais mais de 500 milhø̄es eram obesos (200 milkōes de homens e 300 milhões de mulheres) ${ }^{10}$. As projecçōes para 2015 indicam que 2,3 biliōes de adultos terăo peso a mais e que mais de 700 milhōes serỉo obesos ${ }^{\text {it! }}$.

O presente estudo resultou de un inquérito realizado em algumas farmácias de regiäo de Lisbon, através qual tentámos caracterizar os hábitos alimentares, hábitos de compra, estatuto sócio-económico dos inquiridos, e relacioná-los por meio de um questionário multidimensional com a prevaléncis da obesidade. Os resultados mostrum que mais de metade da populaçâo, utente das farmácias onde se realizou o estudo, é obesa ou evidencia sobrepeso. A prevalência da obesidade e sobrepeso encontrada neste estudo, estí de acordo com o estudo mais recente sobre prevaléncia da obesidade em Portugal, publicado por Isabel 
numbers published in Portugal, by Isabel do Carmo et al. in $2005^{\text {[1! }}$.

Association of educational level and BMI, corroborates other findings from developed countries ${ }^{[2,4,9,}$ showing that less education and lower income are closely related with obesity, since the $26.6 \%$ of subjects with formal education up to the 2 nd cycle were overweight, $13.7 \%$ obese and $0.8 \%$ have shown morbid obesity. The lowest social consumers often prefer low nutritional value diets; highly caloric but also cheaper as demonstrated by Adam Drewnowski and al [a]. Regarding the food price acquisitions choices, data shows that fish, meat, dairy products and fresh fruit and vegetables are the most conditioned by the price. Fish and vegetables are actually among the group of foods that have suffered a greater price increase, while candy, saturated oils and soda are the most price-stable, which obviously has a strong negative impact on the obesity and overweight control ${ }^{[\mathrm{s}]}$.

Regarding the influence of working timetables in meals, we've observed that higher income families, as well as younger people, tends to choose to eat out more often.

\section{Conclusion}

There is still a lot of work to developed on the obesity control and prevention, and this study, despite its nonrepresentative character, draws our attention to some aspects that will certainly determine the portuguese reality. Improvement of the socio-economical condition is one of these aspects since, as demonstrated even in this small study population, these are always the groups prone to obesity and overweight. Fighting against this epidemic must necessarily focus social inequalities and youngsters education, in order to achieve an informed prevention aiming to better and therefore more balanced feeding

\section{Acknowledgements}

The authors express their thanks to all the involved students. Their highly professional performance and dedication were essential to the study. do Carmo e col. em 2005 $5^{\mathrm{rn}}$.

A associação entre o grau de escolaridade e o IMC é também evidente, observando-se que quanto mais baixo é o grau de escolaridade mais elevado é o IMC. Dos $26.6 \%$ dos inquiridos com escolaridade até ao $2^{\circ}$ ciclo com sobrepeso, $13.7 \%$ såo obesos e $0.8 \%$ apresentam obesidade mórbida, o que sugere que, quanto muis baixo é o grau de escolaridade mais elevado é o IMC, dados que estâo de acordo com outros já publicados [21,46,9]. Os grupos socialmente mais desfavorecidos preferem normalmente dietas de baixo valor nutricional, mas com elevado valor calórico, mas que sã̃ contudo de preço mais baixo, como já foi demonstrado ${ }^{\text {[t] }}$.

Quando analisamos as escolhas dos alimentos face ao preço, os dados mostram que os alimentos que são mais condicionados pelo preço sĩo o peixe, a carne, os lacticinios, a fruta $\mathrm{e}$ os legumes frescos. Foi demonstrado que o peixe os legumes frescos estão entre o grupo de alimentos que vêm sofrendo um maior aumento de preço, enquanto que os doces, os óleos saturados e os refrigerantes sĩo os que menos vêm aumentando, o que tem um impacto negativo no controlo da obesidade e sobrepeso ${ }^{|4|}$.

No que diz respeito, à influência que o horário de trabalho exerce nas refeições dos inquiridos foi possivel observar de que aqueles que possuem um maior rendimento familiar e uma faixa etária mais baixa sioo os que afirmar realizar mais refeições fora de casa.

\section{Conclusão}

Existe, ainda muito trabalho a fazer em relação ao controlo e prevençũo da obesidade, e este estudo, apesar do seu carícter nẵo representativo, chama a atenção para alguns aspectos que, certamente, condicionam a realidade portuguesa. A melhoria do condicionante sócio-económico é um destes aspectos jả que, como se demonstra, são os grupos sócioeconómicos mais desfavorecidos aqueles que, mesmo nesta amostra restrita, apresentam uma incidência mais elevada de obesidade e sobrepeso. $\mathrm{O}$ combate a esta epidemia tem necessariamente que atender ao problema das desigualdades sociais, promovendo a educação dos mais jovens, para uma prevenção que vise uma alimentação mais informada e portanto, mais equilibrada.

\section{Agradecimentos}

Os autores expressam os seus agradecimentos aos alunos intervenientes. Graças ao seu profissionalismo e dedicação foi possivel realizar o estudo.

\section{References / Referências}

11. World Health Organizatice. Health in the Context ff Sustainsble Development. Werid Heallh Organization. Osla 2001.

[2]. Cearro I, Santos O, Vieira L, Carreira M, Madina L, Reis L, Myatt J, Teles Galsta A. "Overweight and obesity in Portugal: nutional prevalence in 2003 . 2005 ." Obesity revitas 9 (2007) : 1t - 19 .

[3]. Close $R$, Scoller $D_{n}$ "The Finsuncial Reality of avereating." J Am College Nutritiec 25 (2006)- 203. 209.

[4]. Drewnoswski A, Dermom N. "The ecoenotnics of ebesity dietary energy density and energy cast."
The American Joumal Of Clinical Nutritice 82 (2005) $2655-735$.

[5]. Finkelstein E, Ruhm C, Kasa K. "Economic causes and conseruences of ebesity." Annu. RE. PublieHealth 26 (2005): 239-57.

[6]. K, Mo Pherson. "Does preventing obesity lend to reduced health-cere costs? PLoS Medicine 5 (2003): 153-84.

[7]. Mackeaboch J, Stirbu I, Roskan A, Seasp M, Menviclle G, Leinsalu M, Kunst A. "Socieconomic Inequalities in Health in 22 European Countries." The New Englan Joemal of Medicine (2010): 2468 81.
[8]. Selanen M, Kejantie E, Osmond C, Fcrsen T, Yliharsila H, Erikssan 3. "Tole of Socicecocomic Indicatans an Develapmess of Obesity frum a Life Course Perspective". Journal of Enviromentsl and Publie Health. Wolume 2009: 1.7.

[9], Mertira P, Santos S, Padrto P, Condeino T, Bessa M, Valente H, Barros R, Teixein V, Mitchell V, Lopes C, Mareiza A. " Food Pattem According to Sociedemagraphics, Physical Activity, Sleeping and Obesity in Partagaese Children". Intemational Journsl of Envimenental Researeh fed Publie Heatlb. Wolume 72010 : 1121-1138.the American Association. 1998. 